\title{
BMJ Open Preoxygenation in difficult airway management: high-flow oxygenation by nasal cannula versus face mask (the PREOPTIDAM study). Protocol for a single-centre randomised study
}

\author{
Mickael Vourc'h, ${ }^{1,2}$ Donatien Huard, ${ }^{1}$ Fanny Feuillet, ${ }^{3}$ Gabrielle Baud, ${ }^{1}$ \\ Arthur Guichoux, ${ }^{1}$ Marielle Surbled, ${ }^{1}$ Melanie Tissot, ${ }^{1}$ Anne Chiffoleau, ${ }^{4}$ \\ Christophe Guitton, ${ }^{5}$ Samir Jaber, ${ }^{6}$ Karim Asehnoune ${ }^{1,2}$
}

To cite: Vourc'h M, Huard D, Feuillet $\mathrm{F}$, et al. Preoxygenation in difficult airway management: high-flow oxygenation by nasal cannula versus face mask (the PREOPTIDAM study). Protocol for a single-centre randomised study. BMJ Open 2019;9:e025909. doi:10.1136/ bmjopen-2018-025909

- Prepublication history for this paper is available online. To view these files, please visit the journal online (http://dx.doi. org/10.1136/bmjopen-2018025909).

Received 24 August 2018 Revised 25 January 2019 Accepted 14 February 2019

D Check for updates

(c) Author(s) (or their employer(s)) 2019. Re-use permitted under CC BY-NC. No commercial re-use. See rights and permissions. Published by BMJ.

For numbered affiliations see end of article.

Correspondence to Professor Karim Asehnoune; karim.asehnoune@chu-nantes. fr

\section{ABSTRACT}

Introduction Although preoxygenation and airway management respond to precise algorithms, difficult intubation (DI) remains a daily challenge in intensive care units and in the operating rooms because of its frequent complications, including hypoxaemia. To prevent desaturation during $\mathrm{DI}$, high-flow oxygenation by nasal cannula (HFNC) could prove beneficial. Indeed, contrary to standard preoxygenation device, it can be held in place throughout the intubation trying to perform apnoeic oxygenation during DI. Hence, recent guidelines recommend HFNC during DI, but its relevance has never been evaluated in this setting in a large randomised study until now.

Methods and analysis The PREOPTIDAM trial is a prospective, single-centre, randomised, controlled study in Nantes University Hospital. In anticipated DI, we hypothesised that HFNC can decrease the incidence of desaturation $\leq 94 \%$ or face mask ventilation from $16 \%$ to $4 \%$ compared with standard device. Using a twosided t-test with a first species risk of $5 \%$ and $80 \%$ power, a total of 186 patients will be included. Using a computer-generated randomisation, with a 1:1 allocation ratio, patients will be randomised to HFNC or face mask preoxygenation. Randomisation will be stratified on intubation sequence: Rapid sequence intubation or awake fibreoptic intubation. The primary objective is to determine whether HFNC is more efficient than standard oxygenation techniques to prevent desaturation $\leq 94 \%$ or face mask ventilation during $\mathrm{DI}$. Intent-to-treat and per-protocol analysis are planned for the primary outcome.

Ethics and dissemination The study project has been approved by an independent ethics committee. Written informed consent will be obtained before study inclusion. Participant recruitment begins in September 2018. Results will be submitted to international peer-reviewed medical journals.

Trial registration number NCT03604120.

\section{INTRODUCTION}

Despite major safety improvement over the last decades, substantial morbidity and

\section{Strengths and limitations of this study}

- PREOPTIDAM is the first prospective, randomised, controlled study evaluating high-flow oxygenation by nasal cannula as a preoxygenation and apnoeic oxygenation device during anticipated difficult intubation (DI) in order to prevent desaturation.

- Broad inclusion criteria and large sample size will support external validity.

- Pragmatic study protocol reflects every day practice and results will be of high clinical relevance.

- As no data are available on the incidence of desaturation $\leq 94 \%$ during anticipated $\mathrm{DI}$, an interim analysis will be carried out to allow re-estimating the sample size to maintain power.

mortality remains in anaesthesia. ${ }^{1}$ Hypoxaemia represented $20 \%$ of these severe adverse events. ${ }^{23}$ Although airway management and preoxygenation sequence respond to precise algorithms, anticipated difficult intubation (DI) remains a daily challenge and a major cause of hypoxaemia during anaesthesia. ${ }^{5}$ Preoxygenation, which consists in fulfilling functional residual capacity with pure oxygen, is the cornerstone of patient safety during intubation. Increasing oxygen reserve is the best way to extend safe apnoea duration and therefore to avoid hypoxaemia and its related complications. Current preoxygenation guidelines suggest performing eight vital capacities or $3 \mathrm{~min}$ of spontaneous breathing with a standard face mask, at $\mathrm{FiO}_{2}=100 \%$ in order to achieve $\mathrm{EtO}_{2}$ of $>90 \%{ }^{4}{ }^{5}$ To reduce desaturation during anticipated DI, two options for airway management can be discussed: rapid sequence intubation (RSI) or awake fibreoptic intubation (FOI). ${ }^{4}$ RSI includes preoxygenation with a standard face 
mask, the administration of hypnotic and neuromuscular blocker with rapid onsets and immediate intubation after mask removal without manual ventilation. RSI aims at (1) minimising the time from induction to intubation to reduce the risk of oxygen desaturation ${ }^{5}$; (2) ensuring a fast recovery of spontaneous breathing when intubation proves impossible with difficult face mask ventilation. FOI, usually performed under local anaesthesia and sedation, is proposed for anticipated 'cannot ventilate' or 'limited mouth-opening' patients. It preserves the patient's spontaneous breathing during intubation to avoid major hypoxaemia in case of difficult airway control. After preoxygenation, a dedicated face mask guarantees continuous oxygenation during the procedure. Whatever the option, and despite well-conducted preoxygenation, DI increases first-attempt failure, long-lasting procedure incidence, and leads to frequent oxygen desaturation. ${ }^{6}$ According to the current guidelines, when the level of pulse oximetry $\left(\mathrm{SpO}_{2}\right)$ drops below $95 \%$, the operator has to interrupt intubation and focus on oxygenation (ie, face mask ventilation). ${ }^{7}$ Nevertheless, face mask ventilation could be difficult or impossible in patients with anticipated DI, and could give rise to gastric insufflation or active gag reflex and provoke vomiting or aspiration. It also often requires deepening anaesthesia, leading to severe hypotension. As a result, to limit face mask ventilation during DI could also reduce adverse events, driving research effort in this field.

High-flow oxygenation by nasal cannulae (HFNC) has been studied in the intensive care unit (ICU) and in the operating room as a preoxygenation device, with controversial results. ${ }^{8-10}$ Recent observational studies have suggested the ability of HFNC to extend safe apnoea time during DI and to be held during FOI. ${ }^{11} 12$ This device can deliver up to $60 \mathrm{~L} / \mathrm{min}$ with an inspired fraction of oxygen of up to $100 \%,{ }^{13}$ and generate a moderate positive supraglottic end expiratory pressure. ${ }^{14}$ HFNC could prove beneficial for anticipated DI, during both the preoxygenation and the intubation ${ }^{15}$ : after preoxygenation for RSI, HFNC makes it possible to hold nasal prongs in place during laryngoscopy, trying to perform apnoeic oxygenation throughout the intubation. During FOI, in a spontaneously breathing patient, the preoxygenation and the oxygenation with standard device require a dedicated operator to apply firmly the mask on the patient's face so as to ensure airtightness which is often poorly tolerated. Moreover, in toothless patients or with a beard, significant leaks around the mask can alter oxygenation. HFNC allows to insert the fibrescope in the patient's nostril to perform intubation while continuing the oxygenation and may be better tolerated.

Considering its theoretical advantages and recent recommendations, ${ }^{5} 7$ HFNC must be assessed during preoxygenation and apnoeic oxygenation during anticipated DI. Up to now, no large randomised study has compared HFNC oxygenation with standard of care. Our objective will be to evaluate HFNC preoxygenation for anticipated DI compared with face mask. We hypothesise that HFNC could reduce oxygen desaturation during the intubation and the need of face mask ventilation accordingly.

\section{METHOD AND ANALYSIS \\ Objectives}

\section{Primary objective}

To compare the effectiveness of HFNC (interventional group) and face mask (standard method) as preoxygenation devices to prevent desaturation during anticipated DI.

\section{Secondary objectives}

To compare the quality of preoxygenation, intubation-related complications and patient's outcome until postanaesthesia care unit (PACU) discharge between groups.

\section{Trial design}

The PREOPTIDAM trial will be a prospective, singlecentre, open-label, randomised controlled study. The randomisation sequence will be computer-generated and stratified on the intubation method (RSI or FOI) according to the attending physician's decision. This study will adhere to the international recommendations for interventional trials.

\section{Study settings}

The study will take place at the Nantes University Hospital, France.

\section{Hypothesis}

We hypothesise that compared with face mask preoxygenation, HFNC could reduce the incidence of desaturation $\leq 94 \%$ or the necessity to use face mask ventilation for rescue oxygenation during anticipated DI.

\section{Participant eligibility and consent}

Trial site investigators will identify consecutive eligible patients from the listed criteria. Eligible patients will receive written and oral information. They will be included after investigators have obtained informed written consent.

\section{Inclusion criteria}

- Adults aged from 18 to 90 years

- One major or two minor criteria of anticipated DI (see below)

- And requiring RSI or FOI.

Anticipated DI criteria derived from international guidelines and recent publications ${ }^{416}{ }^{17}$ :

- One major criterion:

- Past DI

- Past laryngeal surgery or radiotherapy

- Limited mouth opening $<25 \mathrm{~mm}$

- Fixed flexion of the cervical spine

- Mallampati IV

- Tumour in the oral or laryngeal region

OR 
- At least two minor criteria:

- Bone to chin distance $<65 \mathrm{~mm}$

- Limited mouth opening $<35 \mathrm{~mm}$ and $>25 \mathrm{~mm}$

- Mallampati III

- Limitation of cervical mobility $\leq 35^{\circ}$

- Neck perimeter $>40 \mathrm{~cm}$ for men and $>38 \mathrm{~cm}$ for women

- Retrognathism

Non-inclusion criteria

- Body mass index $>35 \mathrm{~kg} / \mathrm{m}^{2}$

- Pulse oximetry $<90 \%$ in ambient air

- Haemodynamic instability

- Protected adult

- Pregnancy

- Lack of consent

- Patient already enrolled in another randomised study to improve preoxygenation quality

\section{Assignment of interventions}

\section{Allocation}

Randomisation will be centralised, web-based and accessible 24 hours a day. The randomisation sequence will be carried out in blocks (1:1 ratio) and stratified according to intubation sequence (RSI or FOI).

\section{Sequence generation}

The randomisation sequence will be generated by a statistician working at the Clinical Research department of Nantes University Hospital and not involved in patient recruitment. The software used to collect the data in the electronic case report form (eCRFs) will automatically allocate patients, thereby ensuring concealment. The physicians and a clinical research nurse and/or clinical research assistant will screen the patients for eligibility.

\section{Blinding}

Blinding of the attending physician and patients to the type of preoxygenation device is not feasible. However, the primary outcome is assessed on the basis of an objective criterion.

\section{Trial intervention}

After written informed consent, the patients will be randomly assigned to (see figure 1: study intervention):

- The intervention group: HFNC preoxygenation for $4 \mathrm{~min}$ set at $60 \mathrm{~L} / \mathrm{min}$ of heated and humidified pure oxygen (fraction of inspired oxygen $100 \%, 37^{\circ} \mathrm{C}$ Optiflow; Fisher \& Paykel Healthcare [FPH], Auckland, New Zealand). Large or medium nasal cannulae will be chosen according to the patient's nostril size to limit air contamination. Throughout the intubation procedure, HFNC will be maintained trying to achieve:

- Continuous oxygenation while the patient will be spontaneously breathing during FOI,

- Or apnoeic oxygenation during laryngoscopy for RSI

- The standard group: preoxygenation for 4 min with a face mask (which size will be adapted to fit the patient and ensure airtightness) connected to an Aisys $\mathrm{CS}^{2}$ ventilation system (General Electric, GE Healthcare, Oy, Finland). In this group, the ventilation system is set with $15 \mathrm{~L} / \mathrm{min}$ of fresh gas, $\mathrm{FiO}_{2}=100 \%$, without inspiratory support or expiratory positive pressure.

- For RSI, the face mask (Economy, Intersurgical, Fontenay Sous Bois, France) will be removed after induction to enable intubation.

- For FOI, the face mask (Fibroxy, VBM, Sulz, Germany) will be kept in place throughout the

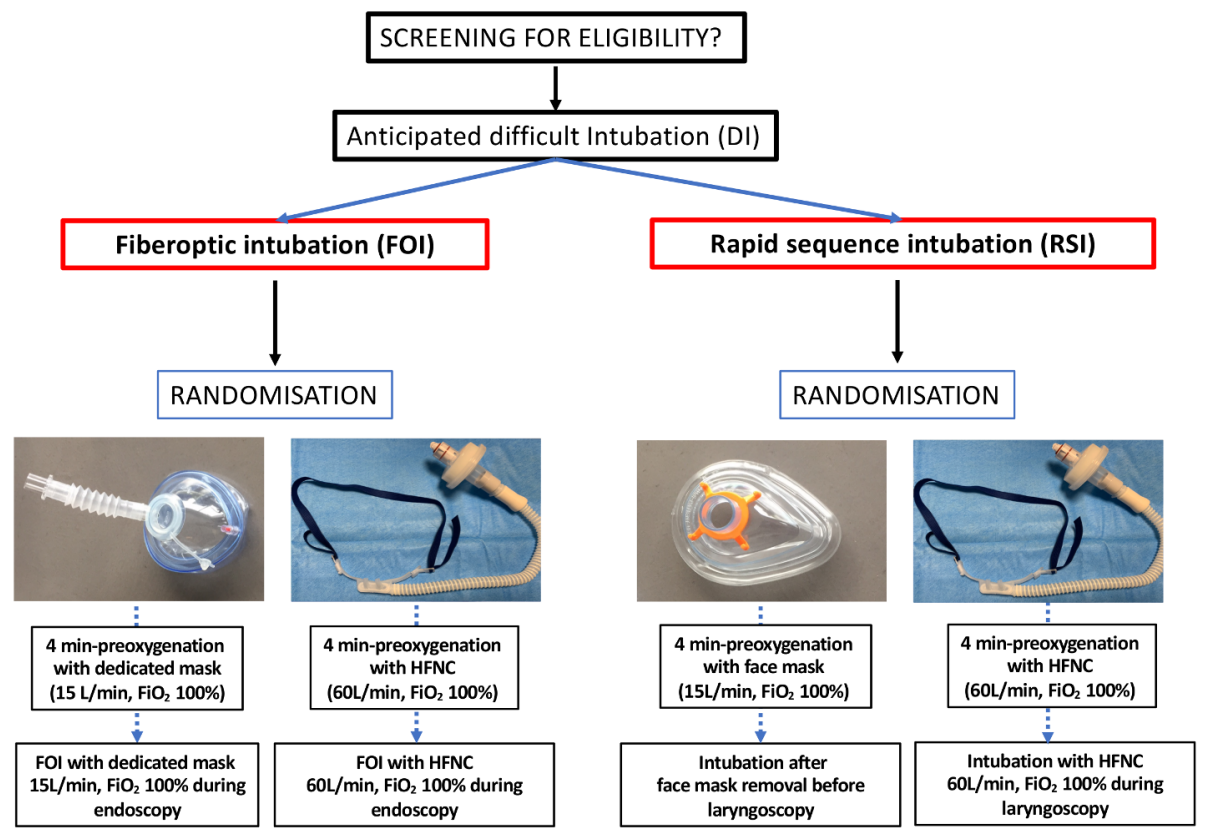

Figure 1 Study design. After attending physician decision to perform RSI or FOI, patients will be randomised to receive highflow nasal cannula (HFNC) or face mask oxygenation. FiO 2, fraction of inspired oxygen. 
intubation procedure with a $15 \mathrm{~L} / \mathrm{min}$ fresh gas flow, $\mathrm{FiO}_{2}=100 \%$, ensuring airtightness.

In both groups, the first operator will be a senior or a junior supervised by a senior. All operators will have assisted a three half-day formation programme so as to be familiar with this three oxygenation devices at the Nantes University Hospital Simulation Centre.

The current guidelines advise to interrupt intubation to focus on oxygenation (ie, face mask ventilation) for oxygen desaturation $\leq 94 \% .{ }^{7}$ Nevertheless, the decision to proceed to face mask ventilation mainly depends on the progression of intubation procedure. Thus, mask ventilation is left at the discretion of the physician as well as the algorithm for rescue oxygenation. However, international recommendation will be presented to all of the investigators before the start of the study. ${ }^{5}$ The attending physician will be free to withdraw the oxygenation device if it disrupts the intubation process or the rescue oxygenation. Clinical data will be collected throughout intubation, surgery and until discharge of the PACU.

\section{Concomitant medication/treatment}

The drugs for sedation and general anaesthesia induction as well as intubation devices will be left to the discretion of the attending physician.

\section{Participant withdrawal}

Patients will be excluded from the trial if they withdraw their consent after randomisation. However, if the patient does not object, the data already collected until consent withdrawal will be analysed. If the patient refuses, the data will be deleted.

\section{Participant timeline and schedule}

Patients will be followed from the beginning of the preoxygenation until PACU discharge (see table 1).

\section{Patient and public involvement}

Patients were not directly involved in the development of the research question or the design of the study. However, the primary and secondary outcomes of the study impact patients' safety and comfort. A written summary of the results of the study will be sent to requesting participants by mail. Participant satisfaction of the intervention will be assessed before PACU discharge and will analysed as a secondary outcome.

\section{Primary and secondary outcome}

Primary outcome

Proportion of patients with desaturation $\leq 94 \%$ or need to use face mask ventilation for oxygen desaturation during intubation in each group.

\section{Measure of the primary outcome}

The patients will be classified in two groups: 'No event' or 'at least one event'.

- For RSI, the primary criterion will be assessed from the induction of general anaesthesia to $2 \mathrm{~min}$ following intubation.

- For FOI, the primary criterion will be assessed from the beginning of sedation to 2 min following intubation.

Arterial oxygen saturation will be evaluated by level of oxygen saturation measured by pulse oximetry $\left(\mathrm{SpO}_{2}\right)$. The evaluation period of $\mathrm{SpO}_{2}$ will be extended to 2 min following intubation completion owing to possible delayed detection of desaturation with this device. Face mask ventilation will be noted if it occurs after general anaesthesia (RSI) or sedation (FOI) induction.

\section{Secondary outcome}

Preoxygenation quality:

- $\mathrm{SpO}_{2}$ at the beginning and at the end of the preoxygenation

- Leaks during preoxygenation defined as:

- In the face mask group: inward or backward leaks with at least $15 \%$ difference between inspired and expired volume.

- In the HFNC group: leaks though the mouth for patients breathing with the mouth opened.

- $\mathrm{EtO}_{2}$ and $\mathrm{EtCO}_{2}$ at the end the preoxygenation (face mask group only)

Intubation procedure until the 2 following minutes:

- Quality of exposure: Cormack-Lehane classification ${ }^{18}$

- Intubation success

- Number of laryngoscopy during RSI

- Number of operators

- Number of alternative devices

Table 1 Participant timeline

\begin{tabular}{lllllll}
\hline & $\begin{array}{l}\text { Anaesthesia } \\
\text { consultation }\end{array}$ & $\begin{array}{l}\text { Preoperative } \\
\text { visit }\end{array}$ & Inclusion & $\begin{array}{l}\text { Intubation and } \\
\text { surgery }\end{array}$ & PACU & PACU discharge \\
\hline Eligibility? & $\mathrm{X}$ & $\mathrm{X}$ & & & & \\
Information & $\mathrm{X}$ & $\mathrm{X}$ & & & \\
Written consent & $\mathrm{X}$ & $\mathrm{X}$ & & & \\
Randomisation & & & $\mathrm{X}$ & $\mathrm{X}$ & \\
Data collection & & & & & $\mathrm{X}$ \\
Exit from the study & & & & & \\
\hline
\end{tabular}

After written informed consent, the patient will be randomised and preoxygenation will be performed according to the allocated device.

Patients will be followed until the postanaesthesia care unit (PACU) discharge. 
- Intubation Difficulty Scale score ${ }^{19}$

- DI rate ${ }^{20}$

- Desaturation $<90 \%$

- Number of episode of face mask ventilation

- Length of intubation procedure from general anaesthesia induction/start of sedation until the end of intubation

- Lowest $\mathrm{SpO}_{2}$

- Lowest $\mathrm{EtO}_{2}$ within 2 min following intubation

- Highest $\mathrm{EtCO}_{2}$ within 2 min following intubation

- Sedation quality during FOI assessed by the "patient sedation score, ${ }^{, 21}$

- Patient's satisfaction score ${ }^{22}$

Intubation-related adverse events during intubation and the following 1 hour:

- Severe complications:

- Death

- Cardiac arrest

- Severe desaturation $<80 \%$

- Severe cardiovascular collapse (systolic blood pressure $<80 \mathrm{~mm} \mathrm{Hg}$ or the need to administer ephedrine or neosynephrine or norepinephrine)

- Mild-to-moderate complications:

- Intubation failure

- Severe ventricular or supraventricular arrhythmia

- Oesophageal intubation

- Dental injury

- Dangerous agitation defined as Richmond Agitation-Sedation Scale $>3$

- Vomiting with aspiration of gastric content

- Nasolaryngotracheal injury or bleeding during RSI or FOI

Per-operative respiratory monitoring:

- Higher $\mathrm{FiO}_{2}$ required to obtain $\mathrm{SpO}_{2}>94 \%$

- Higher plateau pressure at $5 \mathrm{~min}, 30 \mathrm{~min}, 1$ hour after intubation

- Higher peak pressure at $5 \mathrm{~min}, 30 \mathrm{~min}, 1$ hour after intubation

- Achievement of recruitment manoeuvres for desaturation $<95 \%$

- Tidal volume reduction owing to peak pressure $>40 \mathrm{~mm} \mathrm{Hg}$

Morbidity in the PACU:

- Nausea or vomiting

- Inspiratory dyspnoea after extubation

- Lowest $\mathrm{SpO}_{2}$ recorded after extubation

- Desaturation $<90 \%$ before or after extubation

- Severe desaturation $<80 \%$ before or after extubation

- Oxygen therapy requirement at PACU discharge

- Length of stay

- Duration of mechanical ventilation.

- Non-invasive ventilation support

- Reintubation for respiratory failure

Safety issues: severe adverse events

Severe adverse events will be immediately declared and analysed by the vigilance unit of research department of the Nantes University Hospital.
Expected intubation-related adverse events are defined as:

- Severe desaturation $<80 \%$.

- Severe cardiovascular collapse.

- Cardiac arrest and death.

All of the expected or unexpected adverse events (occurring from the beginning of the preoxygenation to the discharge of the PACU) will be collected in the eCRF. The trial may be temporarily stopped for an individual patient at the discretion of the attending physician if a severe adverse event is suspected to be associated with the allocated device.

\section{Statistics analysis and sample size calculation}

The primary outcome is the occurrence (yes or no) of desaturation $\leq 94 \%$ or face mask ventilation during the intubation procedure and the 2 following minutes.

We hypothesise that HFNC can decrease the incidence of desaturation $\leq 94 \%$ or the need of face mask ventilation during intubation from $16 \%$ to $4 \%$ compared with the standard preoxygenation device. The incidence of desaturation during anticipated DI has not been described specifically in the literature up to now. We hypothesise a $12 \%$ reduction in desaturation with HFNC based on our previous study in the ICU (2019, Intensive care medicine, in press). Using a two-sided t-test with a first species risk of $5 \%$ and $80 \%$ power and considering $5 \%$ of consent withdrawal, we planned to include 186 patients. We anticipated approximately 50 patients with FOI indication.

For the primary outcome: a logistic model will compare the incidence of desaturation $\leq 94 \%$ or face mask ventilation. Intent-to-treat and per-protocol analysis are planned for the primary outcome. We shall also perform exploratory subgroup analysis for the primary outcome regarding the type of intubation (FOI or RSI). An interim analysis will be performed after inclusion of half of the total number of patients (93 patients) to enable re-estimation of the sample size to maintain power. ${ }^{23} 24$ The overall probability of the event will be estimated from the pooled data of both treatment groups. If necessary, the sample size will be adjusted accordingly and an amendment to the protocol will be made.

The secondary outcomes will be described and compared between the two groups with linear regression models, generalised mix models or survival models (Cox or Fine and Gray) according to the nature of the variable. All of the analyses will be adjusted to intubation sequence (FOI or RSI).

A predefined statistical analysis plan will be followed using SAS software V.9.3 (Cary, North Carolina, USA). The statistical analysis will incorporate all of the elements required by the CONSORT statement for non-pharmacological interventions.

\section{Track record}

Data will be recorded in a web-based eCRF by the research team. Characteristics at baseline will be gathered: age, sex, weight, height, medical history, indication for 
surgery, predictive criteria of difficult mask ventilation or of DI, description of the intubation procedure (technical aspect, drugs and adverse events). During surgery, respiratory and cardiovascular parameters will be assessed.

Any protocol deviations will be recorded in the eCRF and the medical records. To preserve the confidentiality of personal information, data will be key-coded using alphanumerical numbers. To minimise missing data, to improve the quality of data collection and tracking, an external assessor will collect the variation of $\mathrm{SpO}_{2}$.

\section{Data statement}

Data set will be available on reasonable request to the corresponding author.

\section{Monitoring}

Monitoring will follow 'Good Clinical Practice principles' and will be performed by the independent promotion department of Nantes University Hospital Research Management Unit.

The following data will be assessed:

- Written consent after oral and written information during the anaesthesia consultation.

- Flow chart filled in for included and excluded patients.

- Trial progress.

- Primary and secondary outcome collection.

- Treatment-related severe adverse events.

The eCRF is a secure, interactive, web-response system provided and managed by the data manager team of research department and biometrics unit of the Nantes University Hospital (Nantes, France). The physicians and a clinical research nurse will ensure compliance with the study protocol and collect the study data in the eCRFs.

\section{Trial status}

A total of 186 patients are expected to be included within 19 months.

June 2018: protocol approval by the Ethics Committee.

September 2018: Start of inclusion.

March 2020: End of inclusion.

We will submit the manuscript during the second half of 2020.

\section{ETHICS AND DISSEMINATION}

\section{Research ethics approval}

The trial will be conducted in compliance with the current version of the Declaration of Helsinki and Good Clinical Practice guidelines. The research project was approved.

The study was registered at http://www.clinicaltrials. gov with trial identification number: NCT03604120 before the first inclusion.

\section{Confidentiality}

The study data will be handled as requested by the French Data Protection Authority (Commission Nationale de l'Informatique et des Libertés). All original records will be kept on file at the trial site for 15 years. The electronic trial database file will be anonymised and kept on file for 15 years.

\section{Conflict of interests}

This study was supported by institutional funds and a grant from FPH that is inferior to $20 \%$ of the total budget (24 000 Euros). FPH did not participate in the study design and will not participate in data collection, analysis and interpretation, nor in the preparation, review approval and decision to submit the manuscript for publication.

\section{Dissemination plan}

The study will be published in an international medical journal.

\section{DISCUSSION}

Among the intubation-related adverse events, hypoxaemia is a life-threatening issue and this complication is mainly encountered during difficult airway management. HFNC presents several theoretical advantages compared with the standard face mask, including the ability to deliver continuous oxygen flow to perform apnoeic oxygenation. Recent expert guidelines have advised the use of such a device to prevent desaturation during DI. ${ }^{57}$ However, its relevance has never been evaluated in a large randomised study. This device could improve patient safety but it must be evaluated before systematic implementation in the airway control algorithm.

\section{Author affiliations}

${ }^{1}$ Service d'Anesthésie-Réanimation, Centre Hospitalier Universitaire de Nantes, Nantes, PAYS-DE-LA-LOIRE, France

2UPRES EA 3826, "Thérapeutiques Cliniques et Expérimentales des infections", IRS2 Nantes Biotech, Centre Hospitalier Universitaire de Nantes, Nantes, PAYS-DELA-LOIRE, France

${ }^{3}$ Methodology and Biostatistics Platform, Research Promotion Department, Centre Hospitalier Universitaire de Nantes, Nantes, PAYS-DE-LA-LOIRE, France

${ }^{4}$ Unité de vigilance des essais cliniques, Centre Hospitalier Universitaire de Nantes, Nantes, PAYS-DE-LA-LOIRE, France

${ }^{5}$ Centre Hospitalier du Mans, Médecine intensive réanimation, Le Mans, France

${ }^{6}$ Service d'Anesthésie-Réanimation, Centre Hospitalier Universitaire de Montpellier, Montpellier, France

Acknowledgements We are grateful to the medical staff, nurses and research staff of the Anaesthesia and Critical Care Departments of Nantes University Hospital. We thank Ludivine Perrier for administrative and logistic support, Coralie Ono dit Biot for creating the eCRF, and Dr Anne Chiffoleau, MD for safety monitoring.

Contributors KA and MV obtained funding. KA, SJ, DH, GB, AG, MS, MT, CG and MV designed the study. FF and AC planned the statistical analysis. KA and MV will have full access to the final trial data set. All authors participated in the writing the manuscript and approved the final version.

Funding The PREOPTIDAM trial is supported by the University Hospital of Nantes and a grant from Fisher \& Paykel Healthcare (FPH) that is inferior to $20 \%$ of the total budget (24 000 Euros).

Competing interests MV reports personal fees from MSD, Pfizer, Baxter, grants from Fischer Paykel, outside the submitted work. SJ reports personal fees from Draeger, Fresenius-Xenios and Fisher Paykel Healthcare, outside the submitted work. KA declares personal fees from Fisher Paykel Healthcare, Baxter, LFB, Fresenius. The other authors declared to have no conflict of interest.

Patient consent for publication Obtained. 
Ethics approval By 11 June 2018, the study had been approved by a central ethics committee (Comité de Protection des Personnes lle-de-France II, Paris, France), reference: 2018-04-04 RIPH2.

Provenance and peer review Not commissioned; externally peer reviewed.

Open access This is an open access article distributed in accordance with the Creative Commons Attribution Non Commercial (CC BY-NC 4.0) license, which permits others to distribute, remix, adapt, build upon this work non-commercially, and license their derivative works on different terms, provided the original work is properly cited, appropriate credit is given, any changes made indicated, and the use is non-commercial. See: http://creativecommons.org/licenses/by-nc/4.0/.

\section{REFERENCES}

1. Steadman J, Catalani B, Sharp C, et al. Life-threatening perioperative anesthetic complications: major issues surrounding perioperative morbidity and mortality. Trauma Surg Acute Care Open 2017;2:e000113.

2. Cook TM, Woodall N, Frerk C, et al. Major complications of airway management in the UK: results of the Fourth National Audit Project of the Royal College of Anaesthetists and the Difficult Airway Society. Part 1: anaesthesia. Br J Anaesth 2011;106:617-31.

3. Cheney FW, Posner KL, Lee LA, et al. Trends in anesthesia-related death and brain damage: A closed claims analysis. Anesthesiology 2006;105:1081-6.

4. Apfelbaum JL, Hagberg CA, Caplan RA, et al. Practice guidelines for management of the difficult airway: an updated report by the American Society of Anesthesiologists Task Force on Management of the Difficult Airway. Anesthesiology 2013;118:251-70.

5. Frerk C, Mitchell VS, McNarry AF, et al. Difficult Airway Society 2015 guidelines for management of unanticipated difficult intubation in adults†. BJA: British Journal of Anaesthesia 2015;115:827-48.

6. Workeneh SA, Gebregzi AH, Denu ZA. Magnitude and Predisposing Factors of Difficult Airway during Induction of General Anaesthesia. Anesthesiol Res Pract 2017;2017:1-6.

7. Langeron O, Bourgain JL, Francon D, et al. Difficult intubation and extubation in adult anaesthesia. Anaesth Crit Care Pain Med 2018;37:552-71.

8. Simon M, Wachs C, Braune S, et al. High-flow nasal cannula versus bag-valve-mask for preoxygenation before intubation in subjects with hypoxemic respiratory failure. Respir Care 2016;61:1160-7.

9. Jaber S, Monnin M, Girard M, et al. Apnoeic oxygenation via highflow nasal cannula oxygen combined with non-invasive ventilation preoxygenation for intubation in hypoxaemic patients in the intensive care unit: the single-centre, blinded, randomised controlled OPTINIV trial. Intensive Care Med 2016;42:1877-87.
10. Vourc'h M, Asfar P, Volteau C, et al. High-flow nasal cannula oxygen during endotracheal intubation in hypoxemic patients: a randomized controlled clinical trial. Intensive Care Med 2015:41:1538-48.

11. Patel A, Nouraei SA. Transnasal Humidified Rapid-Insufflation Ventilatory Exchange (THRIVE): a physiological method of increasing apnoea time in patients with difficult airways. Anaesthesia 2015;70:323-9.

12. Badiger S, John M, Fearnley RA, et al. Optimizing oxygenation and intubation conditions during awake fibre-optic intubation using a high-flow nasal oxygen-delivery system. Br J Anaesth 2015;115:629-32.

13. Roca O, Riera J, Torres F, et al. High-flow oxygen therapy in acute respiratory failure. Respir Care 2010;55:408-13.

14. Vargas F, Saint-Leger M, Boyer A, et al. Physiologic effects of highflow nasal cannula oxygen in critical care subjects. Respir Care 2015;60:1369-76.

15. Russotto V, Cortegiani A, Raineri SM, et al. Respiratory support techniques to avoid desaturation in critically ill patients requiring endotracheal intubation: A systematic review and meta-analysis. $J$ Crit Care 2017;41:98-106.

16. Roth D, Pace NL, Lee A, et al. Airway physical examination tests for detection of difficult airway management in apparently normal adult patients. Cochrane Database Syst Rev 2018;5:CD008874.

17. Lundstrøm LH, Møller AM, Rosenstock C, et al. A documented previous difficult tracheal intubation as a prognostic test for a subsequent difficult tracheal intubation in adults. Anaesthesia 2009;64:1081-8.

18. Cormack RS, revisited C-LclassificationBr J Anaesth 2010;105:867-8.

19. Adnet F, Borron SW, Racine SX, et al. The intubation difficulty scale (IDS): proposal and evaluation of a new score characterizing the complexity of endotracheal intubation. Anesthesiology 1997;87:1290-7.

20. American Society of Anesthesiologists Task Force on Management of the Difficult Airway. Practice guidelines for management of the difficult airway: an updated report by the American Society of Anesthesiologists Task Force on Management of the Difficult Airway. Anesthesiology 2003;98:1269-77.

21. Rai MR, Parry TM, Dombrovskis A, et al. Remifentanil targetcontrolled infusion vs propofol target-controlled infusion for conscious sedation for awake fibreoptic intubation: a double-blinded randomized controlled trial. Br J Anaesth 2008;100:125-30.

22. Dhasmana SC. Nasotracheal fiberoptic intubation: patient comfort, intubating conditions and hemodynamic stability during conscious sedation with different doses of dexmedetomidine. J Maxillofac Oral Surg 2014;13:53-8.

23. Friede T, Kieser M. Sample size recalculation for binary data in internal pilot study designs. Pharm Stat 2004;3:269-79.

24. Friede T, Kieser M. Sample size recalculation in internal pilot study designs: a review. Biom J 2006;48:537-55. 